Article

\title{
Effect of Body Mass Index in Coronary CT Angiography Performed on a 256-Slice Multi-Detector CT Scanner
}

\author{
Wei-Yip Law ${ }^{1}$, Guan-Lin Huang ${ }^{2}$ and Ching-Ching Yang ${ }^{3,4, *(\mathbb{D})}$ \\ 1 Department of Radiology, Shin Kong Wu Ho-Su Memorial Hospital, Taipei 111, Taiwan; g000@ms.skh.org.tw \\ 2 Department of Nuclear Medicine, Mennonite Christian Hospital, Hualien 970, Taiwan; \\ s10525006@ems.tcust.edu.tw \\ 3 Department of Medical Imaging and Radiological Sciences, Kaohsiung Medical University, \\ Kaohsiung 807, Taiwan \\ 4 Department of Medical Research, Kaohsiung Medical University Chung-Ho Memorial Hospital, \\ Kaohsiung 807, Taiwan \\ * Correspondence: cyang@kmu.edu.tw
}

Citation: Law, W.-Y.; Huang, G.-L.; Yang, C.-C. Effect of Body Mass Index in Coronary CT Angiography Performed on a 256-Slice

Multi-Detector CT Scanner.

Diagnostics 2022, 12, 319.

https://doi.org/10.3390/ diagnostics12020319

Academic Editor: Jan Fedacko

Received: 28 December 2021

Accepted: 24 January 2022

Published: 27 January 2022

Publisher's Note: MDPI stays neutral with regard to jurisdictional claims in published maps and institutional affiliations.

Copyright: (C) 2022 by the authors. Licensee MDPI, Basel, Switzerland. This article is an open access article distributed under the terms and conditions of the Creative Commons Attribution (CC BY) license (https:// creativecommons.org/licenses/by/ $4.0 /)$.

\begin{abstract}
We aimed to investigate the effect of a patient's body mass index (BMI) on radiation dose and image quality in prospectively ECG-triggered coronary CT angiography (CCTA) performed on a 256-slice multi-detector CT scanner. In total, 87 consecutive patients receiving CCTA examinations acquired with tube current modulation (TCM) and iterative reconstruction (IR) were enrolled in this study. The dose report recorded from the CT scanner console was used to derive the effective dose for patients. Subjective image quality scoring and objective noise measurements were conducted to quantify the impact of BMI on the image quality of CCTA. Because of the TCM technique, we expected tube current and radiation dose to increase as BMI increased. However, using TCM did not always guarantee sufficient radiation exposure to achieve consistent image quality for overweight or obese patients since the maximum X-ray tube output in milliamperes and kilovoltage peak was reached. The impact of photon starvation noise on image quality was not significant until BMI $\geq 27 \mathrm{~kg} / \mathrm{m}^{2}$; this result could be due to IR's noise reduction capability. Our results also suggest that using TCM with a noise index of $25 \mathrm{HU}$ can reduce radiation dose without compromising image quality compared to images obtained based on the manufacturer's default settings.
\end{abstract}

Keywords: CCTA; BMI; radiation dose; image quality

\section{Introduction}

Obesity is a global epidemic, and its prevalence continues to increase in many countries. According to the World Health Organization (WHO) criteria [1], a body mass index (BMI) of $18.5-24.9 \mathrm{~kg} / \mathrm{m}^{2}$ is considered normal weight, while a BMI of $25-29.9 \mathrm{~kg} / \mathrm{m}^{2}$ is considered overweight or pre-obese. A BMI $\geq 30 \mathrm{~kg} / \mathrm{m}^{2}$ is defined as obese, and the obese category can be sub-divided into obese class I (30-34.9 kg/m²), obese class II $\left(35-39.9 \mathrm{~kg} / \mathrm{m}^{2}\right)$, and obese class III $\left(\geq 40 \mathrm{~kg} / \mathrm{m}^{2}\right)$. Obesity is a significant risk factor for several subtypes of cardiovascular diseases, such as coronary heart disease, stroke, and heart failure [2-4]. Coronary CT angiography (CCTA) is widely recognized as an important imaging tool in the diagnosis of coronary artery disease. However, high photon attenuation and scatter both affect the image quality of CCTA scans of obese patients. The tube current modulation (TCM) system automatically adapts X-ray output according to a patient's shape, size, and attenuation to achieve a specified image quality with the goal of providing adequate image quality at the lowest possible radiation dose [5-7]. In addition to TCM, iterative reconstruction (IR) also has the ability to reduce photon starvation noise resulting from patient attenuation. IR methods are developed to reduce image noise by performing a recursive search for the best estimate to provide an image quality at higher noise levels similar to images acquired by conventional filtered back projection (FBP) [8-11]. Equivalent 
quality between images with a dose reduction of $44-54 \%$ and FBP images has been reported with the application of IR in CCTA [12-14]. Because TCM and IR methods have good dose efficiency to achieve desired image quality, they may prove beneficial to improving the diagnostic accuracy of CCTA by reducing the photon starvation noise that is due to patient attenuation. The aim of this study was to investigate the image quality and radiation dose of prospectively ECG-triggered CCTA acquired with TCM and IR performed on a 256-slice multi-detector CT (MDCT) scanner for patients with BMIs $<25$ and $\geq 25 \mathrm{~kg} / \mathrm{m}^{2}$.

\section{Materials and Methods}

\subsection{Study Population}

A total of 87 consecutive CCTA examinations for adult patients (26 women and 61 men; age range: 31-82 years; heart rate (HR) range: 45-106 bpm; and BMI range: $18.21-38.19 \mathrm{~kg} / \mathrm{m}^{2}$ ) undergoing prospectively ECG-triggered axial scans were investigated in this study. Exclusion criteria for CCTA included known iodine allergy, impaired renal function, and pregnancy. Patients were referred because of chest pain (16\%), dyspnea (7\%), positive stress test $(10 \%)$, elevated cardiovascular risk $(3 \%)$, coronary stent $(3 \%)$, physical exam (50\%), and follow-up (11\%). Approval was gained from the Institutional Human Research Ethics Committee to obtain access to patient data.

\subsection{CCTA Acquisition}

Patients with an HR > 70 bpm were given 10-30 mg of oral propranolol (Propranolol Tab, Standard Chem \& Pharm Co., Ltd., Tainan, Taiwan) one hour before examination. All patients received $0.6 \mathrm{mg}$ of sublingual nitroglycerin (Nitrostat, Pfizer Pharma, Vega Baja, Puerto Rico) 2 min prior to the scan unless contraindicated. CCTA acquisition was performed using prospectively ECG-triggered axial scanning on a 256-row detector CT scanner (Revolution CT, GE Healthcare, Milwaukee, WI, USA). Non-ionic contrast agent (Optiray 350, loversol injection 74\%, Liebel-Flarsheim Canada Inc., Pointe-Claire, QC, Canada) was administered intravenously in a dose of $0.8 \mathrm{~mL} / \mathrm{kg}$ body weight at a flow rate of $3.5-6.0 \mathrm{~mL} / \mathrm{s}$, followed by a $30 \mathrm{~mL}$ saline chaser at 3.0-5.0 mL/s. CCTA scans were triggered automatically by a delay of $5.9 \mathrm{~s}$ when contrast enhancement within the descending aorta reached a threshold level of 80 Hounsfield units (HU). The scan field of view (SFOV) and z-axis coverage were chosen according to the heart size. The ECG pulsing window was set at 70-85\% of the R-R interval for patients with an $\mathrm{HR} \leq 65 \mathrm{bpm}$. For patients with $65 \mathrm{bpm}<\mathrm{HR} \leq 85 \mathrm{bpm}$, full tube current was applied at 40-55\% and $70-85 \%$ of the R-R interval. For patients with an HR $>85 \mathrm{bpm}$, the ECG pulsing window was set at $20-90 \%$ of the R-R interval. The tube voltage was adjusted manually based on patient attenuation, while the tube current was determined automatically by a TCM system (SmartmA, GE Healthcare, Milwaukee, WI, USA) to achieve a noise index (NI) of $22 \mathrm{HU}$. After scanning, images were reconstructed with 50\% FBP and ASiR-V blending, a slice thickness of $0.625 \mathrm{~mm}$, and a $512 \times 512$ matrix size. Axial images, volume rendering images, and curved planar reformation images were comprehensively evaluated. A cardiac motion correction algorithm (SnapShot Freeze, GE Healthcare, Milwaukee, WI, USA) was used during reconstruction to reduce coronary motion blurring.

\subsection{Image Quality Evaluation}

The image quality of CCTA scans was evaluated by conducting subjective and objective image quality assessments $[15,16]$.

Subjective image quality was rated using a 4-point grading scale for quantifying the impact of photon starvation noise on every coronary artery segment with a diameter of at least $1.0 \mathrm{~mm}$ : 1 = excellent (excellent attenuation of the vessel lumen and clear delineation of the vessel walls with limited perceived image noise); 2 = good (impact of image noise, limitations of low contrast resolution, and minimal vessel margin definition); 3 = adequate (reduced image quality with poor vessel wall definition or excessive image noise, and limitations of low-contrast resolution remained evident); 4 = poor (impaired 
image quality limited by excessive noise or poor vessel wall definition). Coronary arteries were classified according to the American Heart Association 15-segment model [17]. The right coronary artery (RCA), the left main and left anterior descending artery (LAD), and the left circumflex artery (LCX) were defined to include segments 1-4, 5-10, and 11-15, respectively. Two experienced cardiovascular radiologists rated images by consensus to quantify the degradation of image quality based on photon starvation noise. The subjective image quality scores rated on a per-segment basis were averaged to give an overall score for each vessel. Examples of CCTA images rated at 1 (excellent), 2 (good), and 3 (adequate) are shown in Figure 1. Because no image was rated at 4 (poor) in this study, an example image is not shown. Please refer to [16] for a CCTA image with poor quality.
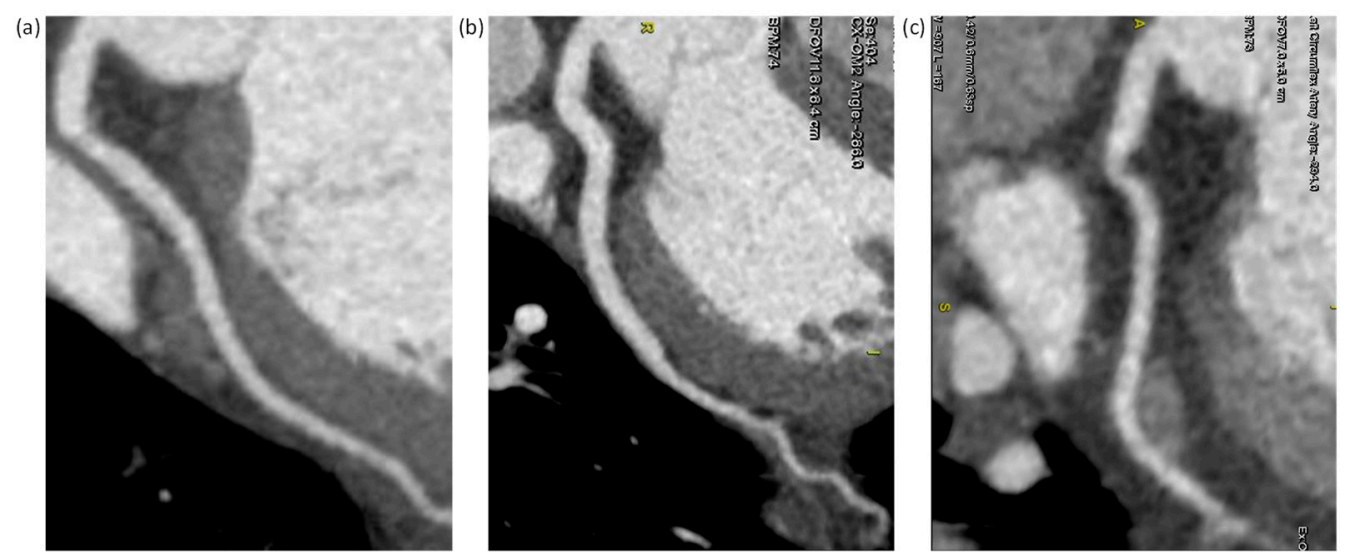

Figure 1. Curved multiplanar reformations of the left circumflex artery (LCX) subjectively rated at 1 , 2, and 3 (left to right). (a) Heart rate $(\mathrm{HR})=61 \mathrm{bpm}$, body mass index $(\mathrm{BMI})=21.8 \mathrm{~kg} / \mathrm{m}^{2}$, and noise index $(\mathrm{NI})=24 \mathrm{HU}$; (b) $\mathrm{HR}=74 \mathrm{bpm}, \mathrm{BMI}=27.7 \mathrm{~kg} / \mathrm{m}^{2}$, and $\mathrm{NI}=27.7 \mathrm{HU}$; (c) $\mathrm{HR}=73 \mathrm{bpm}$, $\mathrm{BMI}=31.1 \mathrm{~kg} / \mathrm{m}^{2}$, and $\mathrm{NI}=30.4 \mathrm{HU}$.

Objective noise measurements expressed as image noise, contrast-to-noise ratio (CNR), and signal-to-noise ratio (SNR) were obtained by evaluation of the region of interest (ROI) in each image. Image noise was obtained by calculating the standard deviation (SD) of the CT numbers within a contrast-filled left ventricle (circular region: $0.2 \mathrm{~cm}^{2}$ ). CNR was calculated as the difference between the mean signal intensity of the contrast-filled left ventricle and the mean signal intensity of the left ventricular posterolateral wall, divided by the image noise defined previously (circular region: $0.2 \mathrm{~cm}^{2}$ ). SNR was calculated as the mean signal intensity of the contrast-enhanced coronary lumen divided by its SD in the proximal segments of the left and right coronary arteries (circular region: $0.02 \mathrm{~cm}^{2}$ ).

\subsection{Radiation Dose}

The volume CT dose index $\left(\mathrm{CTDI}_{\mathrm{vol}}\right)$ and the dose-length product (DLP) displayed on the scanner console were recorded after CCTA scans. The effective dose was calculated as the product of DLP times a conversion coefficient for the chest $\left(\mathrm{k}=0.014 \mathrm{mSv} \times[\mathrm{mGy} \times \mathrm{cm}]^{-1}\right)[18]$.

\subsection{Statistical Analysis}

Student's t-test was used to compare numerical data, including age, BMI, HR, HR variability, tube voltage, tube current, exposure time, SFOV, $z$-axis coverage, $\mathrm{CTDI}_{\mathrm{vol}}$, DLP, effective dose, contrast dose, image noise, CNR, and SNR. For the subjective image quality scores, which are ordinal data, comparisons were performed using the Mann-Whitney $\mathrm{U}$ test. A statistically significant difference was defined as a two-sided $p$-value $<0.05$. The inter-observer agreement for subjective image quality scores was analyzed with the kappa test $(\kappa<0.40$ : poor agreement; $0.40 \leq \kappa<0.75$ : good agreement; and $\kappa \geq 0.75$ : excellent agreement). Statistical analysis was performed using SPSS version 18 (IBM, Armonk, New York, NY, USA). 


\section{Results}

A total of 87 patients were enrolled in this study, including 45 patients with BMIs $<25 \mathrm{~kg} / \mathrm{m}^{2}$ and 42 patients with BMIs $\geq 25 \mathrm{~kg} / \mathrm{m}^{2}$. Detailed patient characteristics, scan parameters, and radiation doses are summarized in Table 1. When comparing these two BMI groups, significant differences were found in age, BMI, tube voltage, tube current, $\mathrm{CTDI}_{\mathrm{vol}}, \mathrm{DLP}_{\text {, }}$ and effective dose.

Table 1. Comparison of patient characteristics, scan parameters, and radiation doses between two BMI groups.

\begin{tabular}{|c|c|c|c|c|}
\hline & & BMI $<25$ & $\mathrm{BMI} \geq 25$ & $p$-Value \\
\hline \multicolumn{2}{|c|}{ Number of patients } & 45 & 42 & - \\
\hline \multicolumn{2}{|c|}{ Age } & $60.80 \pm 8.75$ & $56.64 \pm 10.67$ & $0.049 *$ \\
\hline \multicolumn{2}{|c|}{$\operatorname{BMI}\left(\mathrm{kg} / \mathrm{m}^{2}\right)$} & $22.74 \pm 1.33$ & $27.93 \pm 2.47$ & $<0.001 *$ \\
\hline \multirow{4}{*}{ SFOV } & small & 0 & 0 & \\
\hline & medium & 39 & 38 & 0.583 \\
\hline & large & 6 & 4 & \\
\hline & $12 \mathrm{~cm}$ & 0 & 2 & \\
\hline \multirow[t]{2}{*}{$Z$-axis coverage } & $14 \mathrm{~cm}$ & 18 & 18 & 0.293 \\
\hline & $16 \mathrm{~cm}$ & 27 & 22 & \\
\hline \multicolumn{2}{|c|}{$\mathrm{HR}(\mathrm{bpm})$} & $65.16 \pm 9.55$ & $62.71 \pm 8.61$ & 0.215 \\
\hline \multicolumn{2}{|c|}{ HR variability (bpm) } & $5.71 \pm 4.72$ & $5.57 \pm 3.28$ & 0.874 \\
\hline \multicolumn{2}{|c|}{ Tube voltage $(\mathrm{kVp})$} & $112.44 \pm 9.81$ & $120.95 \pm 6.17$ & $<0.001 *$ \\
\hline \multicolumn{2}{|c|}{ Tube current (mA) } & $637.38 \pm 92.17$ & $701.67 \pm 39.94$ & $<0.001 *$ \\
\hline \multicolumn{2}{|c|}{ Exposure time (s) } & $0.74 \pm 0.09$ & $0.71 \pm 0.11$ & 0.259 \\
\hline \multicolumn{2}{|c|}{$\mathrm{CTDI}_{\mathrm{vol}}(\mathrm{mGy})$} & $20.03 \pm 6.22$ & $24.83 \pm 5.78$ & $<0.001 *$ \\
\hline \multicolumn{2}{|c|}{$\operatorname{DLP}(\mathrm{mGy} \times \mathrm{cm})$} & $303.49 \pm 96.21$ & $372.89 \pm 90.84$ & $0.001 *$ \\
\hline \multicolumn{2}{|c|}{ Effective dose (mSv) } & $4.25 \pm 1.35$ & $5.22 \pm 1.27$ & $0.001^{*}$ \\
\hline
\end{tabular}

* Statistically significant difference was found between the 2 BMI groups. Note: BMI = body mass index $\mathrm{SFOV}=$ scan field of view, $\mathrm{HR}=$ heart rate, $\mathrm{CTDI}_{\mathrm{vol}}=$ volume CT dose index, DLP: dose-length product .

Figure 2 shows the optimal reconstruction phase for the RCA, LAD, and LCX. There were 1098 coronary artery segments in 87 patients, which included 338 RCA segments, 491 LAD segments, and 269 LCX segments. Systolic and diastolic reconstruction were used in $20.41 \%$ and $79.59 \%$ of the RCA segments, respectively (BMI $<25 \mathrm{~kg} / \mathrm{m}^{2}$ : 38 segments vs. 140 segments; $B M I \geq 25 \mathrm{~kg} / \mathrm{m}^{2}$ : 31 segments vs. 129 segments). The corresponding results were $7.74 \%$ and $92.26 \%$ for $\mathrm{LAD}$ segments (BMI $<25 \mathrm{~kg} / \mathrm{m}^{2}$ : 24 segments vs. 233 segments; $\mathrm{BMI} \geq 25 \mathrm{~kg} / \mathrm{m}^{2}$ : 14 segments vs. 220 segments) and $7.06 \%$ and $92.94 \%$ for LCX segments (BMI $<25 \mathrm{~kg} / \mathrm{m}^{2}$ : 13 segments vs. 124 segments; BMI $\geq 25 \mathrm{~kg} / \mathrm{m}^{2}$ : 6 segments vs. 126 segments).

Table 2 summarizes the contrast agent doses, scan parameters, and radiation doses in different BMI groups. These baseline characteristics significantly increased for BMIs of $25-26,27-28$, and $>28 \mathrm{~kg} / \mathrm{m}^{2}$ compared to a BMI $<23 \mathrm{~kg} / \mathrm{m}^{2}$. Figure 3 demonstrates the distribution of tube voltage, tube current, and $\mathrm{CTDI}_{\mathrm{vol}}$ as the functions of BMI.

Table 3 summarizes the NI, subjective image quality scores, and objective noise measurements in different BMI groups. The inter-observer agreement for subjective scores was excellent (kappa $=0.92 ; 95 \%$ confidence interval: 0.86-0.98). Through evaluating these image quality indicators, we found that image noise increased as BMI increased. In comparison with a BMI $<23 \mathrm{~kg} / \mathrm{m}^{2}$, NI was significantly higher in BMIs of 25-26, $27-28$, and $>28 \mathrm{~kg} / \mathrm{m}^{2}$, while subjective scores significantly degraded in BMIs of 27-28 and $>28 \mathrm{~kg} / \mathrm{m}^{2}$. For objective noise measurements, statistically significant differences were found in CNR $\left(\mathrm{BMI}>28 \mathrm{~kg} / \mathrm{m}^{2}\right)$ and SNR of the left and right coronary arteries $\left(\mathrm{SNR}_{\mathrm{L}}\right.$ : BMI $>28 \mathrm{~kg} / \mathrm{m}^{2} ; \mathrm{SNR}_{\mathrm{R}}$ : BMIs of $25-26,>28 \mathrm{~kg} / \mathrm{m}^{2}$ ) when compared to a BMI $<23 \mathrm{~kg} / \mathrm{m}^{2}$, but not for image noise. Figure 4 demonstrates the subjective scores for the RCA, LAD, and LCX as the functions of BMI. Figure 5 demonstrates the $C N R, S_{L}$, and $S_{R} R_{R}$ as functions of BMI. Case examples with a subjective score of two are shown in Figure 6. 

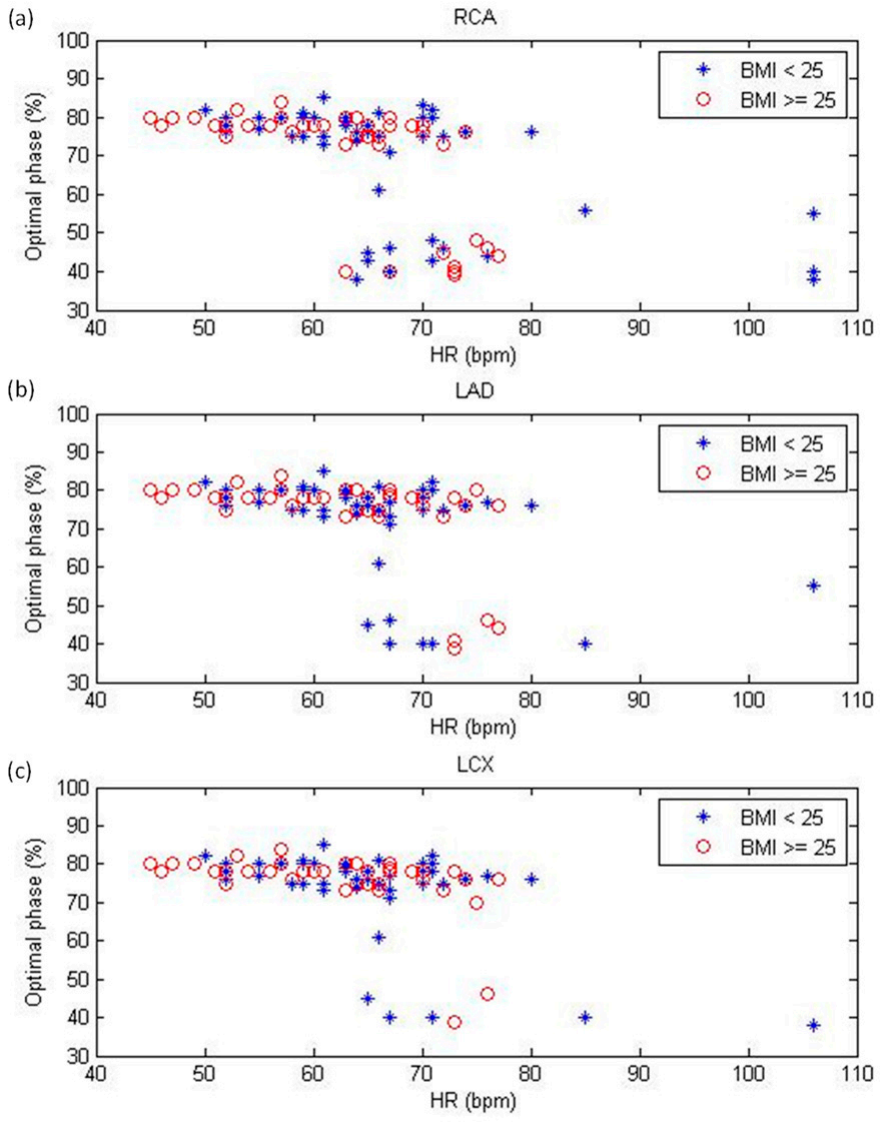

Figure 2. Optimal reconstruction phase for the (a) right coronary artery (RCA), (b) left anterior descending artery (LAD), and (c) left circumflex artery (LCX) as functions of heart rate (HR) on a per-segment basis. $\mathrm{BMI}=$ body mass index.

Table 2. Comparison of contrast agent doses, scan parameters, and radiation doses in different BMI groups.

\begin{tabular}{|c|c|c|c|c|c|c|c|}
\hline & BMI $\left(\mathrm{kg} / \mathrm{m}^{2}\right)$ & $\begin{array}{c}\text { Number of } \\
\text { Patients }\end{array}$ & $\begin{array}{c}\text { Contrast } \\
\text { Agent }(\mathrm{mL})\end{array}$ & $\begin{array}{c}\text { Tube Voltage } \\
(\mathbf{k V p})\end{array}$ & $\begin{array}{c}\text { Tube Current } \\
(\mathrm{mA})\end{array}$ & $\begin{array}{l}\mathrm{CTDI}_{\mathrm{vol}} \\
\text { (mGy) }\end{array}$ & $\begin{array}{c}\text { Effective } \\
\text { Dose }(\mathrm{mSv})\end{array}$ \\
\hline \multirow[b]{2}{*}{$\mathrm{BMI}<25$} & $<23$ & 25 & 55.28 & 111.20 & 627.56 & 19.42 & 4.13 \\
\hline & $23-24$ & 20 & $\begin{array}{c}60.45 \\
(p=0.180)\end{array}$ & $\begin{array}{c}114.00 \\
(p=0.347)\end{array}$ & $\begin{array}{c}649.65 \\
(p=0.431)\end{array}$ & $\begin{array}{c}20.80 \\
(p=0.467)\end{array}$ & $\begin{array}{c}4.40 \\
(p=0.506)\end{array}$ \\
\hline \multirow{3}{*}{$\mathrm{BMI} \geq 25$} & $25-26$ & 17 & $\begin{array}{c}68.47^{*} \\
(p<0.001)\end{array}$ & $\begin{array}{c}118.82 * \\
(p=0.002)\end{array}$ & $\begin{array}{c}707.82 * \\
(p=0.001)\end{array}$ & $\begin{array}{c}24.32 * \\
(p=0.022)\end{array}$ & $\begin{array}{c}5.09 * \\
(p=0.040)\end{array}$ \\
\hline & $27-28$ & 14 & $\begin{array}{c}73.14^{*} \\
(p<0.001)\end{array}$ & $\begin{array}{c}121.43^{*} \\
(p<0.001)\end{array}$ & $\begin{array}{c}705.71 * \\
(p=0.001)\end{array}$ & $\begin{array}{c}25.10^{*} \\
(p=0.012)\end{array}$ & $\begin{array}{c}5.20 * \\
(p=0.031)\end{array}$ \\
\hline & $>28$ & 11 & $\begin{array}{c}82.36^{*} \\
(p<0.001)\end{array}$ & $\begin{array}{c}123.64 * \\
(p=0.001)\end{array}$ & $\begin{array}{c}687.00 * \\
(p=0.02)\end{array}$ & $\begin{array}{c}25.27^{*} \\
(p=0.026)\end{array}$ & $\begin{array}{c}5.44 * \\
(p=0.021)\end{array}$ \\
\hline
\end{tabular}

* Statistically significant difference was found when compared to $\mathrm{BMI}<23 \mathrm{~kg} / \mathrm{m}^{2}$. Note: $\mathrm{BMI}=$ body mass index $\mathrm{CTDI}_{\mathrm{vol}}=$ volume $\mathrm{CT}$ dose index . 

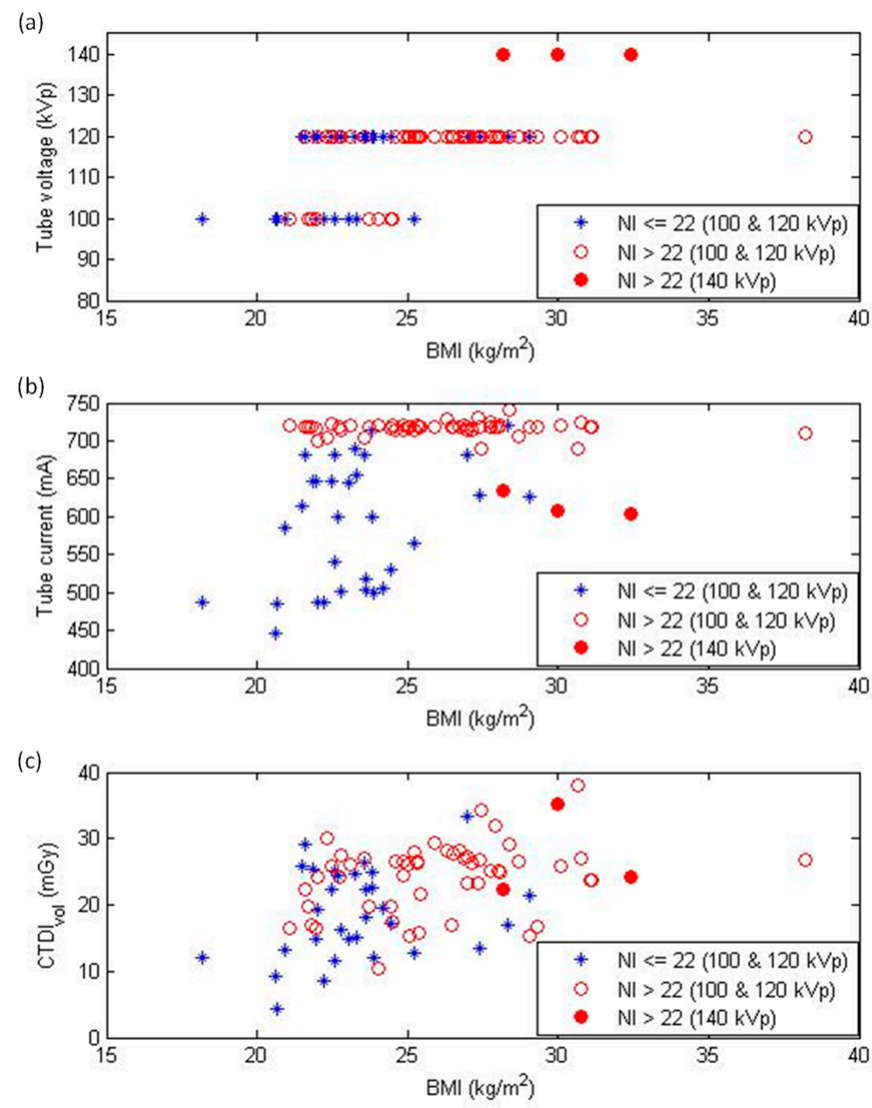

Figure 3. Distribution of (a) tube voltage, (b) tube current, and (c) volume CT dose index $\left(\mathrm{CTDI}_{\mathrm{vol}}\right)$ as functions of body mass index (BMI). NI = noise index.

Table 3. Comparison of NI, subjective image quality score, and objective noise measurements in different BMI groups.

\begin{tabular}{|c|c|c|c|c|c|c|c|c|c|c|}
\hline & \multirow{2}{*}{$\begin{array}{c}\text { BMI } \\
\left(\mathrm{kg} / \mathrm{m}^{2}\right)\end{array}$} & \multirow{2}{*}{$\begin{array}{l}\text { Number } \\
\text { of Patients }\end{array}$} & \multirow{2}{*}{$\begin{array}{c}\text { NI } \\
\text { (HU) }\end{array}$} & \multicolumn{3}{|c|}{ Noise Score } & \multirow{2}{*}{$\begin{array}{l}\text { Image Noise } \\
\text { (HU) }\end{array}$} & \multirow{2}{*}{ CNR } & \multirow{2}{*}{$\mathrm{SNR}_{\mathrm{L}}$} & \multirow{2}{*}{$\mathrm{SNR}_{\mathrm{R}}$} \\
\hline & & & & RCA & LAD & LCX & & & & \\
\hline \multirow[b]{2}{*}{$\mathrm{BMI}<25$} & $<23$ & 25 & 22.82 & 1.73 & 1.66 & 1.67 & 26.40 & 16.57 & 25.15 & 25.44 \\
\hline & $23-24$ & 20 & $\begin{array}{c}23.56 \\
(p=0.237)\end{array}$ & $\begin{array}{c}1.75 \\
(p=0.689)\end{array}$ & $\begin{array}{c}1.57 \\
(p=0.808)\end{array}$ & $\begin{array}{c}1.58 \\
(p=0.735)\end{array}$ & $\begin{array}{c}27.57 \\
(p=0.636)\end{array}$ & $\begin{array}{c}15.19 \\
(p=0.346)\end{array}$ & $\begin{array}{c}24.00 \\
(p=0.779)\end{array}$ & $\begin{array}{c}25.49 \\
(p=0.990)\end{array}$ \\
\hline \multirow{3}{*}{$\mathrm{BMI} \geq 25$} & $25-26$ & 17 & $\begin{array}{c}25.68 * \\
(p<0.001)\end{array}$ & $\begin{array}{c}1.76 \\
(p=0.460)\end{array}$ & $\begin{array}{c}1.65 \\
(p=0.739)\end{array}$ & $\begin{array}{c}1.63 \\
(p=0.755)\end{array}$ & $\begin{array}{c}29.45 \\
(p=0.272)\end{array}$ & $\begin{array}{c}14.24 \\
(p=0.148)\end{array}$ & $\begin{array}{c}19.92 \\
(p=0.080)\end{array}$ & $\begin{array}{c}18.16^{*} \\
(p=0.018)\end{array}$ \\
\hline & $27-28$ & 14 & $\begin{array}{c}28.11^{*} \\
(p<0.001)\end{array}$ & $\begin{array}{c}2.18^{*} \\
(p=0.002)\end{array}$ & $\begin{array}{c}2.05^{*} \\
(p=0.006)\end{array}$ & $\begin{array}{c}2.07^{*} \\
(p=0.005)\end{array}$ & $\begin{array}{c}27.88 \\
(p=0.628)\end{array}$ & $\begin{array}{c}15.59 \\
(p=0.582)\end{array}$ & $\begin{array}{c}28.77 \\
(p=0.348)\end{array}$ & $\begin{array}{c}27.82 \\
(p=0.564)\end{array}$ \\
\hline & $>28$ & 11 & $\begin{array}{c}30.64^{*} \\
(p=0.002)\end{array}$ & $\begin{array}{c}2.18^{*} \\
(p=0.011)\end{array}$ & $\begin{array}{c}2.09 * \\
(p=0.008)\end{array}$ & $\begin{array}{c}2.171 * \\
(p=0.014)\end{array}$ & $\begin{array}{c}30.93 \\
(p=0.133)\end{array}$ & $\begin{array}{c}12.79 * \\
(p=0.033)\end{array}$ & $\begin{array}{c}16.29 * \\
(p=0.004)\end{array}$ & $\begin{array}{c}18.46^{*} \\
(p=0.028)\end{array}$ \\
\hline
\end{tabular}

*: Statistically significant difference was found compared to BMI $<23 \mathrm{~kg} / \mathrm{m}^{2}$. Note: BMI $=$ body mass index $\mathrm{NI}=$ noise index, $\mathrm{RCA}=$ right coronary artery, $\mathrm{LAD}=$ left anterior descending artery, $\mathrm{LCX}=$ left circumflex artery $\mathrm{CNR}=$ contrast-to-noise ratio, $\mathrm{SNR}_{\mathrm{L}}=$ signal-to-noise ratio of left coronary artery, $\mathrm{SNR}_{\mathrm{R}}=$ signal-to-noise ratio of right coronary artery. 

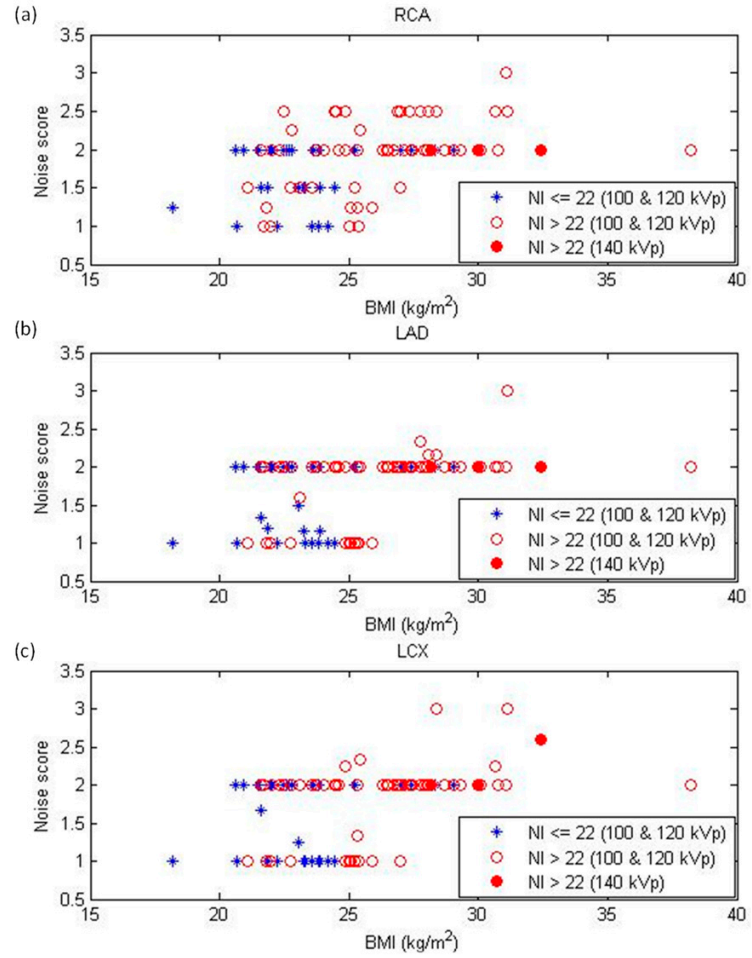

Figure 4. Subjective noise score on a per-vessel basis for the (a) right coronary artery (RCA), (b) left anterior descending artery (LAD), and (c) left circumflex artery (LCX) as functions of body mass index (BMI). $\mathrm{NI}=$ noise index.

(a)

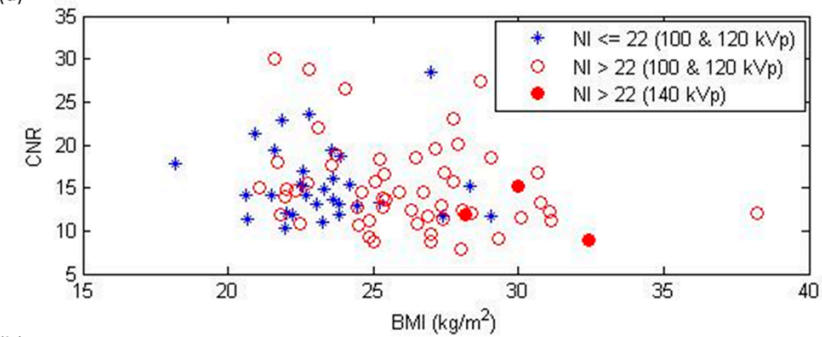

(b)
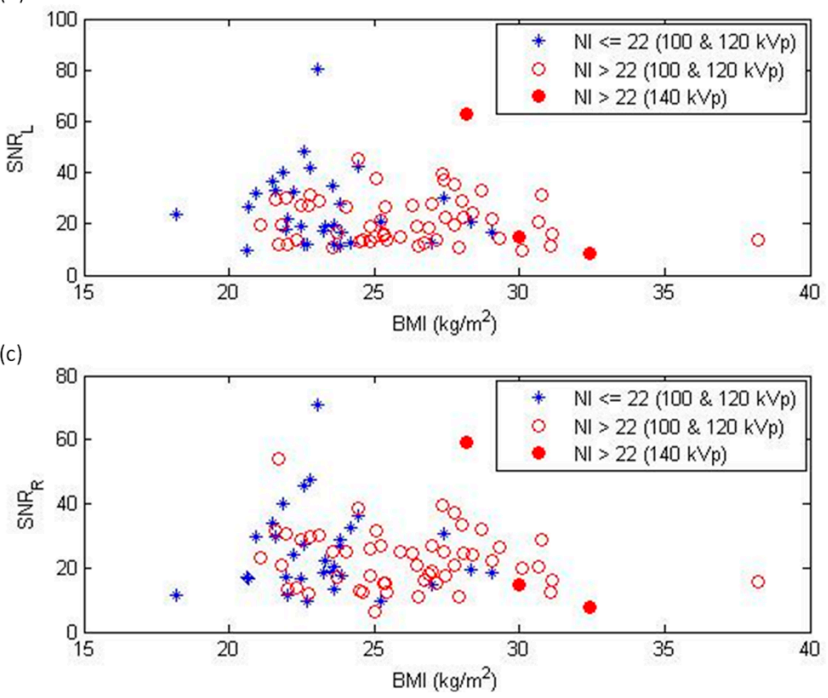

Figure 5. Objective image quality expressed in terms of (a) contrast-to-noise ratio (CNR), (b) signal-to-noise ratio of left coronary artery $\left(\mathrm{SNR}_{\mathrm{L}}\right)$, and (c) signal-to-noise ratio of right coronary artery $\left(\mathrm{SNR}_{\mathrm{R}}\right)$ as functions of body mass index $(\mathrm{BMI})$. $\mathrm{NI}=$ noise index. 

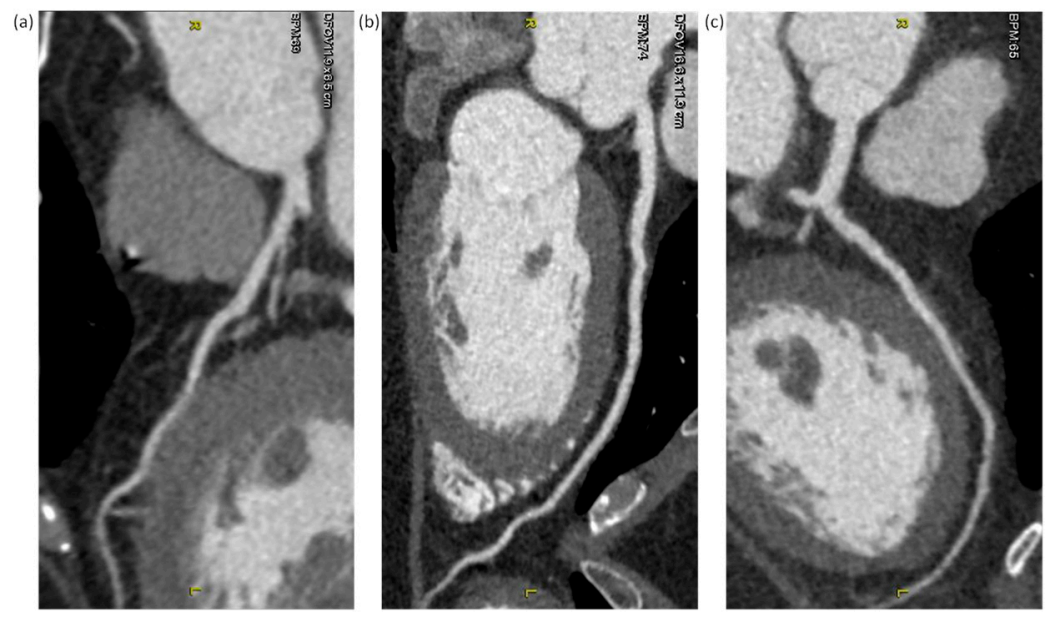

Figure 6. Curved multiplanar reformations of the left anterior descending artery (LAD) subjectively rated at 2 . (a) Heart rate $(\mathrm{HR})=69 \mathrm{bpm}$, body mass index $(\mathrm{BMI})=21.6 \mathrm{~kg} / \mathrm{m}^{2}$, and noise index $(\mathrm{NI})=22.7 \mathrm{HU}$; (b) $\mathrm{HR}=74 \mathrm{bpm}, \mathrm{BMI}=27.4 \mathrm{~kg} / \mathrm{m}^{2}$, and $\mathrm{NI}=27.7 \mathrm{HU}$; (c) $\mathrm{HR}=65 \mathrm{bpm}$, $\mathrm{BMI}=38.2 \mathrm{~kg} / \mathrm{m}^{2}$, and $\mathrm{NI}=38.8 \mathrm{HU}$.

\section{Discussion}

With increasing patient thickness, photon attenuation increases exponentially [19]. The resultant artifacts are worse with the use of a low-power generator system, low peak tube voltage, and fast rotation time. To provide consistent image quality for all patients, the TCM technique was used to compensate for the photon starvation noise that is due to patient attenuation. In the TCM system we operated, the NI, or the predefined image quality index, was set at $22 \mathrm{HU}$, according to the manufacturer's recommendation. However, an NI $>22 \mathrm{HU}$ indicated that the photon starvation noise was not fully compensated for at this setting. Therefore, we selected a proper tube voltage setting before data acquisition to achieve the desired noise level according to the NI displayed on the console. In this study, CCTA scans were acquired at 100 or $120 \mathrm{kVp}$ for BMI $<25 \mathrm{~kg} / \mathrm{m}^{2}$, while a tube voltage of 120 or $140 \mathrm{kVp}$ was used for BMI $\geq 25 \mathrm{~kg} / \mathrm{m}^{2}$ (Figure 3a). Based on our experience, the difference between 120 and $140 \mathrm{kVp}$ CCTA scans when imaging obese patients is small in terms of radiation dose and image noise (Figures 3-5). This phenomenon occurs because the maximum tube current is lower at $140 \mathrm{kVp}$ than at $120 \mathrm{kVp}$. For the CT scanner investigated in this study, the maximum tube current was $740 \mathrm{~mA}$ at 100 and $120 \mathrm{kVp}$, while the maximum tube current was $635 \mathrm{~mA}$ at $140 \mathrm{kVp}$. Additionally, prior studies have shown that the iodine contrast attenuation is lower in CCTA images acquired with a higher kilovoltage peak [20-22], so the $140 \mathrm{kVp}$ scan is usually not used in our clinical routine practice.

TCM adjusts the tube current based on the patient attenuation in the scout image to achieve the desired image quality at the lowest dose setting, so there should be a positive correlation between tube current and patient attenuation. However, the positive correlation between tube current and patient attenuation reached a plateau beyond the maximum X-ray output in milliamperes and kilovoltage peak, and the efficacy of TCM on compensating photon starvation noise was limited in the plateau region. As shown in Table 1, we found that kilovoltage peak, milliamperes, $\mathrm{CTDI}_{\mathrm{vol}}$, DLP, and effective dose are significantly higher for BMI $\geq 25 \mathrm{~kg} / \mathrm{m}^{2}$. A more detailed comparison shown in Table 2 reveals that these baseline characteristics increased as BMI increased, except the tube current for a BMI of $27-28 \mathrm{k}$ and $>28 \mathrm{~kg} / \mathrm{m}^{2}$. As seen in Figure $3 \mathrm{~b}$, there was a positive correlation between tube current and BMI for CCTA scans with an NI $\leq 22 \mathrm{HU}$ (blue stars). However, an increase in BMI did not lead to obvious variation in the tube current of CCTA scans with an NI $>22 \mathrm{HU}$ (red circles). There were $40.0 \%$ and $88.1 \%$ CCTA scans with an NI $>22 \mathrm{HU}$ for BMI $<25$ and $\geq 25 \mathrm{~kg} / \mathrm{m}^{2}$, respectively. Because of the small size and rapid motion of the coronary arteries, CCTA requires high spatial resolution (small voxel size) and 
high temporal resolution (short rotation time) to achieve reliable diagnostic image quality. Consequently, the TCM-adjusted tube current is usually higher for CCTA than in other CT studies, which makes it easier to reach the maximum $X$-ray output when imaging obese patients. In addition to the TCM modulation range, the photon starvation noise cannot be fully eliminated, and the noise level increases with increasing BMI. Therefore, the CCTA scans acquired using the maximum X-ray output have an NI value larger than $22 \mathrm{HU}$.

Since the tube voltage, tube current, and radiation doses increased as BMI increased (Table 2 and Figure 3), it is natural to expect that these CCTA scans would provide consistent image quality for all patients. However, we found that the subjective image quality scores and objective noise measurements only slightly increased with increasing BMI (Table 3, Figure 4). This phenomenon could be due to the CCTA scans with an NI > $22 \mathrm{HU}$. The resulting impact was more serious for patients with BMI $\geq 25 \mathrm{~kg} / \mathrm{m}^{2}$. Although our results demonstrated a positive correlation between image quality degradation and BMI, the overall subjective score of each vessel was less than three, indicating the image quality was sufficient for clinical diagnosis. As seen in Table 3, the difference between $\mathrm{BMI}<23$ and BMI $25-26 \mathrm{~kg} / \mathrm{m}^{2}$ was statistically significant in NI, but not in subjective scoring or objective noise measurements. These results indicated that NI is more sensitive to the variation in BMI than other image quality indicators. By definition, NI is the noise in the central region of a CT image reconstructed with FBP for a $20 \mathrm{~cm}$ uniform water phantom [23]. The subjective scoring and objective noise measurements were performed on IR-reconstructed images. IR yields lower image noise and higher spatial resolution than FBP, which could explain why the subjective scoring and objective noise measurements were less sensitive to the increased image noise that is due to patient attenuation compared to NI. Moreover, the subjective scoring and objective noise measurements were defined for contrast-filled vessels and chambers, while NI was defined on a phantom-simulating soft tissue. It was reported that image noise has a larger detrimental effect on the detection of a low-contrast target than it does on detection of a high-contrast target [24]. Therefore, the definition of image quality metrics might be another potential explanation of the differing sensitivity to image noise.

In this study, the $\mathrm{CTDI}_{\mathrm{vol}}$ for $\mathrm{BMI}<25$ and $\geq 25 \mathrm{~kg} / \mathrm{m}^{2}$ were 20.03 and $24.83 \mathrm{mGy}$, respectively, which are consistent with previous reports $[25,26]$. Over the past decades, various dose reduction strategies have been proposed for CCTA scans [27,28]. Some are general techniques (such as prospective ECG triggering, low kilovoltage peak, and TCM) and others are technology-specific techniques (such as hybrid/statistical IR, model-based IR, and deep learning IR). Although minimizing the radiation dose is always an important goal in the CT imaging community, it is also important to ensure sufficient image quality for clinical diagnosis at the same time, i.e., the as low as reasonably achievable (ALARA) principle. Since there was no significant difference in the subjective image quality scores and objective noise measurements between BMI $<23$ and $25-26 \mathrm{~kg} / \mathrm{m}^{2}$, we expected that using an NI of $25 \mathrm{HU}$ could reduce the radiation dose without compromising the image quality for patients receiving CCTA performed on the 256-slice MDCT investigated in this study. Several limitations to this study need to be acknowledged. First, we relied on data that already existed in our department. To avoid selection bias, data were enrolled consecutively during a specific time period, and image analysis was performed without information about BMI. Second, since we focused on the effect of BMI on the radiation dose and image noise of CCTA, the diagnostic performance of CCTA was not compared to invasive catheter angiography. Further studies are necessary to evaluate the impact of BMI on the diagnostic assessment of the coronary arteries with CT. Third, all data were acquired using TCM and IR developed by a single manufacturer. It was difficult to compare our results with other types of TCM and IR techniques from different manufacturers.

\section{Conclusions}

In this study, we investigated the effect of a patient's BMI on the radiation dose and image quality in prospectively ECG-triggered CCTA performed on a 256-slice MDCT. 
Because of the TCM technique, we expected that the tube current and radiation dose would increase as BMI increased. However, using TCM could not always guarantee sufficient radiation exposure to achieve consistent image quality for overweight or obese patients since the maximum X-ray tube output in milliamperes and kilovoltage peak was reached. The impact of photon starvation noise on image quality was not significant until $\mathrm{BMI} \geq 27 \mathrm{~kg} / \mathrm{m}^{2}$, which could have been due to IR's noise reduction capability. Our results also suggest that using TCM with an NI of $25 \mathrm{HU}$ could reduce the radiation dose without compromising image quality compared to images obtained based on the manufacturer's default settings.

Author Contributions: Conceptualization, W.-Y.L. and C.-C.Y.; methodology, W.-Y.L., G.-L.H. and C.-C.Y.; software, G.-L.H. and C.-C.Y.; validation, W.-Y.L. and C.-C.Y.; formal analysis, G.-L.H. and C.-C.Y.; investigation, G.-L.H. and C.-C.Y.; resources, W.-Y.L. and C.-C.Y.; data curation, W.-Y.L. and C.-C.Y.; writing—original draft preparation, C.-C.Y.; writing—review and editing, C.-C.Y.; visualization, W.-Y.L., G.-L.H. and C.-C.Y.; supervision, C.-C.Y.; project administration, C.-C.Y. All authors have read and agreed to the published version of the manuscript.

Funding: This research was supported in part by a grant from the Ministry of Science and Technology in Taiwan (MOST110-2314-B-037-076).

Institutional Review Board Statement: The study was conducted according to the guidelines of the Declaration of Helsinki, and approved by the Institutional Review Board of Kaohsiung Medical University Chung-Ho Memorial Hospital (protocol code KMUHIRB-E(II)-20210003; approved on 19 January 2021).

Informed Consent Statement: Patient consent was waived by the Institutional Review Board, because this retrospective study represents no more than minimal risk to subjects and did not adversely affect their rights and welfare.

Data Availability Statement: The authors confirm that the data supporting the findings of this study are available within the article.

Conflicts of Interest: The authors declare no conflict of interest.

\section{References}

1. World Health Organization. Obesity: Preventing and Managing the Global Epidemic; Report of a WHO Consultation; Technical Report Series 894; World Health Organization: Geneva, Switzerland, 2000; 253p.

2. Benjamin, E.J.; Blaha, M.J.; Chiuve, S.E.; Cushman, M.; Das, S.R.; Deo, R.; De Ferranti, S.D.; Floyd, J.; Fornage, M.; Gillespie, C.; et al. Heart Disease and Stroke Statistics-2017 Update: A Report from the American Heart Association. Circulation 2017, 135, e146-e603. [CrossRef]

3. Forouzanfar, M.H.; Alexander, L.; Anderson, H.R.; Bachman, V.F.; Biryukov, S.; Brauer, M.; Burnett, R.; Casey, D.; Coates, M.M.; Cohen, A.; et al. Global, regional, and national comparative risk assessment of 79 behavioural, environmental and occupational, and metabolic risks or clusters of risks in 188 countries, 1990-2013: A systematic analysis for the Global Burden of Disease Study 2013. Lancet 2015, 386, 2287-2323. [CrossRef]

4. Roth, G.A.; Huffman, M.D.; Moran, A.E.; Feigin, V.; Mensah, G.A.; Naghavi, M.; Murray, C.J. Global and regional patterns in cardiovascular mortality from 1990 to 2013. Circulation 2015, 132, 1667-1678. [CrossRef]

5. MacDougall, R.D.; Kleinman, P.L.; Callahan, M.J. Size-based protocol optimization using automatic tube current modulation and automatic kV selection in computed tomography. J. Appl. Clin. Med. Phys. 2016, 17, 328-341. [CrossRef]

6. Soderberg, M.; Gunnarsson, M. Automatic exposure control in computed tomography-an evaluation of systems from different manufacturers. Acta Radiol. 2010, 51, 625-634. [CrossRef]

7. Schindera, S.T.; Nelson, R.C.; Yoshizumi, T.; Toncheva, G.; Nguyen, G.; DeLong, D.M.; Szucs-Farkas, Z. Effect of automatic tube current modulation on radiation dose and image quality for low tube voltage multidetector row CT angiography: Phantom study. Acad. Radiol. 2009, 16, 997-1002. [CrossRef]

8. Den Harder, A.M.; Willemink, M.J.; De Ruiter, Q.M.; De Jong, P.A.; Schilham, A.M.; Krestin, G.P.; Leiner, T.; Budde, R.P. Dose reduction with iterative reconstruction for coronary CT angiography: A systematic review and meta-analysis. Br. J. Radiol. 2016, 89, 20150068. [CrossRef]

9. Aschoff, A.J.; Catalano, C.; Kirchin, M.A.; Krix, M.; Albrecht, T. Low radiation dose in computed tomography: The role of iodine. Br. J. Radiol. 2017, 90, 20170079. [CrossRef] 
10. Wang, R.; Schoepf, U.J.; Wu, R.; Nance, J.W., Jr.; Lv, B.; Yang, H.; Li, F.; Lu, D.; Zhang, Z. Diagnostic accuracy of coronary CT angiography: Comparison of filtered back projection and iterative reconstruction with different strengths. J. Comput. Assist. Tomogr. 2014, 38, 179-184. [CrossRef]

11. Buls, N.; Van Gompel, G.; Van Cauteren, T.; Nieboer, K.; Willekens, I.; Verfaillie, G.; Evans, P.; Macholl, S.; Newton, B.; De Mey, J. Contrast agent and radiation dose reduction in abdominal CT by a combination of low tube voltage and advanced image reconstruction algorithms. Eur. Radiol. 2015, 25, 1023-1031. [CrossRef]

12. Klink, T.; Obmann, V.; Heverhagen, J.; Stork, A.; Adam, G.; Begemann, P. Reducing CT radiation dose with iterative reconstruction algorithms: The influence of scan and reconstruction parameters on image quality and CTDIvol. Eur. J. Radiol. 2014, 83, 1645-1654. [CrossRef] [PubMed]

13. Padole, A.; Ali Khawaja, R.D.; Kalra, M.K.; Singh, S. CT radiation dose and iterative reconstruction techniques. AJR Am. J. Roentgenol. 2015, 204, W384-W392. [CrossRef] [PubMed]

14. Geyer, L.L.; Schoepf, U.J.; Meinel, F.G.; Nance, J.W., Jr.; Bastarrika, G.; Leipsic, J.A.; Paul, N.S.; Rengo, M.; Laghi, A.; De Cecco, C.N State of the Art: Iterative CT Reconstruction Techniques. Radiology 2015, 276, 339-357. [CrossRef] [PubMed]

15. Mangold, S.; Wichmann, J.L.; Schoepf, U.J.; Litwin, S.E.; Canstein, C.; Varga-Szemes, A.; Muscogiuri, G.; Fuller, S.R.; Stubenrauch, A.C.; Nikolaou, K.; et al. Coronary CT angiography in obese patients using 3(rd) generation dual-source CT: Effect of body mass index on image quality. Eur. Radiol. 2016, 26, 2937-2946. [CrossRef]

16. Wong, D.T.; Soh, S.Y.; Ko, B.S.; Cameron, J.D.; Crossett, M.; Nasis, A.; Troupis, J.; Meredith, I.T.; Seneviratne, S.K. Superior CT coronary angiography image quality at lower radiation exposure with second generation 320-detector row CT in patients with elevated heart rate: A comparison with first generation 320-detector row CT. Cardiovasc. Diagn. Ther. 2014, 4, 299-306. [CrossRef]

17. Austen, W.G.; Edwards, J.E.; Frye, R.L.; Gensini, G.G.; Gott, V.L.; Griffith, L.S.; McGoon, D.C.; Murphy, M.L.; Roe, B.B. A reporting system on patients evaluated for coronary artery disease. Report of the Ad Hoc Committee for Grading of Coronary Artery Disease, Council on Cardiovascular Surgery, American Heart Association. Circulation 1975, 51, 5-40. [CrossRef]

18. Deak, P.D.; Smal, Y.; Kalender, W.A. Multisection CT protocols: Sex- and age-specific conversion factors used to determine effective dose from dose-length product. Radiology 2010, 257, 158-166. [CrossRef]

19. Modica, M.J.; Kanal, K.M.; Gunn, M.L. The obese emergency patient: Imaging challenges and solutions. Radiographics 2011, 31, 811-823. [CrossRef]

20. Van Hamersvelt, R.W.; Eijsvoogel, N.G.; Mihl, C.; De Jong, P.A.; Schilham, A.M.R.; Buls, N.; Das, M.; Leiner, T.; Willemink, M.J. Contrast agent concentration optimization in CTA using low tube voltage and dual-energy CT in multiple vendors: A phantom study. Int. J. Cardiovasc. Imaging 2018, 34, 1265-1275. [CrossRef]

21. Scholtz, J.E.; Ghoshhajra, B. Advances in cardiac CT contrast injection and acquisition protocols. Cardiovasc. Diagn. Ther. 2017, 7, 439-451. [CrossRef]

22. Wang, Y.; Bolen, M.; Sydow, G.; Kottha, A.; Soufan, K.; Bullen, J.; Kemper, C.; Kalafut, J.; Halliburton, S.S. Adaptation of contrast injection protocol to tube potential for cardiovascular CT. AJR Am. J. Roentgenol. 2014, 203, 1181-1191. [CrossRef] [PubMed]

23. Gudjonsdottir, J.; Ween, B.; Olsen, D.R. Optimal use of AEC in CT: A literature review. Radiol. Technol. 2010, 81, $309-317$.

24. Goenka, A.H.; Herts, B.R.; Obuchowski, N.A.; Primak, A.N.; Dong, F.; Karim, W.; Baker, M.E. Effect of reduced radiation exposure and iterative reconstruction on detection of low-contrast low-attenuation lesions in an anthropomorphic liver phantom: An 18-reader study. Radiology 2014, 272, 154-163. [CrossRef]

25. Stocker, T.J.; Deseive, S.; Leipsic, J.; Hadamitzky, M.; Chen, M.Y.; Rubinshtein, R.; Heckner, M.; Bax, J.J.; Fang, X.-M.; Grove, E.L.; et al. Reduction in radiation exposure in cardiovascular computed tomography imaging: Results from the PROspective multicenter registry on radiaTion dose Estimates of cardiac CT angIOgraphy iN daily practice in 2017 (PROTECTION VI). Eur. Heart J. 2018, 39, 3715-3723. [CrossRef]

26. Hamilton-Craig, C.R.; Tandon, K.; Kwan, B.; DeBoni, K.; Burley, C.; Wesley, A.J.; O’Rourke, R.; Neill, J.; Branch, K.R. Coronary CT radiation dose reduction strategies at an Australian Tertiary Care Center-Improvements in radiation exposure through an evidence-based approach. J. Med. Radiat. Sci. 2020, 67, 25-33. [CrossRef]

27. Hedgire, S.S.; Baliyan, V.; Ghoshhajra, B.B.; Kalra, M.K. Recent advances in cardiac computed tomography dose reduction strategies: A review of scientific evidence and technical developments. J. Med. Imaging 2017, 4, 031211. [CrossRef]

28. Litmanovich, D.E.; Tack, D.M.; Shahrzad, M.; Bankier, A.A. Dose reduction in cardiothoracic CT: Review of currently available methods. Radiographics 2014, 34, 1469-1489. [CrossRef] 